\title{
LEES' IMMERSION THEOREM AND THE TRIANGULATION OF MANIFOLDS
}

\author{
BY R. LASHOF ${ }^{1}$
}

Communicated by William Browder, December 9, 1968

In [4] Lees proves the following immersion theorem for topological manifolds: Let $M, M^{\prime}, Q$ be topological manifolds, $M$ a compact locally flat submanifold of the open manifold $M^{\prime}$, with $\operatorname{dim} M^{\prime}$ $=\operatorname{dim} Q=q$, and $\partial Q=\varnothing$. Write $\operatorname{Im}_{M^{\prime}}(M, Q)$ for the s.s. complex of $M^{\prime}$ immersions of $M$ in $Q$; and write $R\left(T M^{\prime} / M, T Q\right)$ for the s.s. complex of representative germs of $T M^{\prime} / M$ in $T Q$. A representative germ is a bundle map of the tangent bundle $T M^{\prime}$ of $M^{\prime}$, restricted to a neighborhood of $M$, into the tangent bundle $T Q$ of $Q$. Two germs are identified if they agree over a common neighborhood of $M$.

TheOREM (LEes). If $M$ has a handle decomposition with all handles of index $<Q$; the differential $d: \operatorname{Im}_{M^{\prime}}(M, Q) \rightarrow R\left(T M^{\prime} / M, T Q\right)$ is a homotopy equivalence.

We show here how to simplify some of the hypotheses of this theorem and give applications to the problem of triangulating topological manifolds.

THEOREM A. In the following two cases, the assumption that $M$ has a handle decomposition may be dropped in Lees' Immersion Theorem.

(1) $\operatorname{dim} M<\operatorname{dim} Q$.

(2) $\operatorname{dim} M=\operatorname{dim} Q \geqq 5$, and $Q$ is a piecewise linear $(P L)$ manifold.

Of course, if $M$ is a PL-manifold, $M$ has a handle decomposition, and hence Lees' theorem applies.

TheOREM B. In the following cases, $R\left(T M^{\prime} / M, T Q\right)$ may be taken to be the s.s. complex of ordinary bundle maps of $T M^{\prime}$, restricted to $M$, into $T Q$.

(1) $\operatorname{dim} M=\operatorname{dim} Q$.

(2) $\operatorname{dim} M<\operatorname{dim} Q, M$ a closed submanifold of $M^{\prime}$ and $M$ the homotopy type of a locally finite simplicial complex.

We will say that an $R^{k}$-bundle $\mathcal{E}$ over a space dominated by a locally finite simplicial complex $K$ admits a PL-bundle structure, if the pullback of $\varepsilon$ over $K$ is the underlying topological bundle of a PL- $R^{k_{-}}$

1 The author was partially supported by an NSF grant. 
bundle over $K$. (This is independent of choice of dominating complex and maps.)

Now let $M$ be closed, $\operatorname{dim} M=n \geqq 5$, and $M$ simply connected. Write $M^{0}=M$-open $n$-ball.

TheOREM C. If the tangent bundle of $M^{0}$ admits a PL-bundle structure, $M$ admits a PL-manifold structure.

Finally we have

Theorem D. Let $M_{1}, M_{2}$ be closed $P L$-manifolds; $\operatorname{dim} M_{i} \geqq 5$, and $M_{i}$ simply connected, $i=1,2$. A homeomorphism $h: M_{1} \rightarrow M_{2}$ is concordant (or weakly isotopic) to a PL-homeomorphism, if and only if the topological bundle map $d h \oplus 1: T\left(M_{1}\right) \oplus 1 \rightarrow T\left(M_{2}\right) \oplus 1$ is homotopic through topological bundle maps to a PL-bundle map.

Proof of A. Since the essential trick in proving (1) is also used in proving (2), we only prove the latter.

We will need

Lees' Lemma. Let $M^{n}$ be a topological manifold (without boundary), $n \geqq 5$; if $M^{n}$-point admits a $P L$-manifold structure, $M^{n}$ admits a $P L$ manifold structure.

Proof of Lemma. By the Novikov-Siebenmann theorem [5], [6], the end of $M^{n}$-point has a neighborhood PL-equivalent to $\Sigma^{n-1} \times R$, $\Sigma^{n-1}$ a PL-homotopy sphere. But for $n \geqq 5, \Sigma^{n-1} \times R$ is PL-equivalent to $S^{n-1} \times R$. By taking $t$ sufficiently large, $S^{n-1} \times t$ is contained in the interior of a disc neighborhood $D^{n}$ of the point in $M^{n}$. By the Shoenflies theorem [2], $S^{n-1} \times t$ bounds a disc $D_{1}^{n}$ in $D^{n}$. Thus ( $\left.M^{n}-\operatorname{Int} D_{1}^{n}\right)$ $\cup C\left(S^{n-1} \times t\right)$ is a PL-manifold homeomorphic to $M^{n}$.

PROof of $\mathrm{A}(2)$. It will be sufficient to show that if either $\operatorname{Im}_{M^{\prime}}(M, Q)$ or $R\left(T M^{\prime} / M, T Q\right)$ is nonempty, there is a neighborhood $V$ of $M$ in $M^{\prime}$ that admits a PL-manifold structure. For then there is a compact PL-manifold $N$, with $M \subset$ Int $N \subset V^{\prime}, V$ any sufficiently small neighborhood of $M$. Since $N$ has a handle decomposition, we may apply Lees' theorem to $N$, and the result follows easily.

Now if $\operatorname{Im}_{M^{\prime}}(M, Q)$ is nonempty, there is an immersion $f: V \rightarrow Q$, $V$ some open neighborhood of $M$. But then $V$ admits a PL-manifold structure, since $Q$ does.

If $R\left(T M^{\prime} / M, T Q\right)$ is nonempty, there is a neighborhood $U$ of $M$ and a bundle map $\psi: T U \rightarrow T Q$. Cover $M$ by a finite number of coordinate neighborhoods $\left\{V_{i}^{\prime}\right\}$ and let $\left\{V_{i}\right\}$ be a shrinking of this cover, with $\bar{V}_{i} \subset V_{i}^{\prime}, \bar{V}_{i}$ compact. Let $C_{t}=\bigcup_{i=1}^{r} \bar{V}_{i}$. We will prove inductively, 
that if $\phi_{r-1}: U_{r-1} \rightarrow Q$ is an immersion of a neighborhood of $C_{r-1}$, such that $d \phi_{r-1}$ is homotopic to $\psi \mid U_{r-1}$, then there is an immersion $\phi_{r}: U_{r}$ $\rightarrow Q$, where $U_{r}$ is a neighborhood of $C_{r}$ and $d \phi_{r} \sim \psi \mid U_{r}$. (The result is trivial for $C_{1}=\bar{V}_{1}$.)

Triangulate $V_{r}^{\prime}$ sufficiently fine, so that any simplex of $V_{r}^{\prime}$ that meets $C_{r-1}$ is contained in $U_{r-1}$. Now $\bar{V}_{r}$ is contained in a finite subcomplex $K$ of $V_{r}^{\prime}$. Now by induction over the skeletons, we can immerse a neighborhood $W$ of $C_{r-1} \cup K^{(k)}$, using Lemma 2 of [4], with $n=k$, provided $k<q$. Since $Q$ is PL, $W$ admits a PL-manifold structure. Thus a neighborhood $W^{\prime}$ of $C_{r}$ admits a PL-structure except at a finite number of points. Therefore, $W^{\prime}$ admits a PL-structure by Lee's lemma. But then there is a compact PL-manifold $N_{r}$, with $C_{r} \subset$ Int $N_{r} \subset W^{\prime}$. By applying Lees' theorem to $N_{r}$ we obtain an immersion $\phi_{r}$ of a neighborhood $U_{r}$ of $N_{r}$ (and hence of $C_{r}$ ) with $d \phi_{r}$ homotopic to $\psi$.

This completes the inductive step, and hence there is an immersion $\phi: U \rightarrow Q, U$ a neighborhood of $M$ in $M^{\prime}$. Hence $U$ admits a PLstructure. Q.E.D.

Proof of B. If $\operatorname{dim} M=\operatorname{dim} Q$, then $M$ has a collar in $M^{\prime}$, and hence is a deformation retract of a neighborhood $U$. It follows that any bundle map of $T M^{\prime} / M$ extends canonically to $T M^{\prime} / U$, and any two such are canonically homotopic relative to $M$. Thus the two definitions of $R\left(T M^{\prime} / M, T Q\right)$ are equivalent.

For $\operatorname{dim} M<\operatorname{dim} Q$, the author does not know whether a locally flat submanifold is a neighborhood deformation retract; however, it is true stably. First note that if $\varepsilon$ is an $R^{n}$-bundle over a space $X$ of the homotopy type of a locally finite simplicial complex, the total space $E(\varepsilon)$ also has this property; and it follows that the projection $p: E(\varepsilon) \rightarrow X$ and zero section $i: X \rightarrow E(\varepsilon)$ are homotopy inverses, and $X$ is a deformation retract of $E(\varepsilon)$.

Now $M$ has a normal bundle $\nu$ in $M^{\prime} \times R^{k}, k$ sufficiently large, and $M$ is a (strong) deformation retract of $E(\nu)$. Since $T M^{\prime}$ may be lifted to a bundle $\tau$ over $M^{\prime} \times R^{k}$ such that $\tau \mid M^{\prime} \times 0=T M^{\prime}$, it follows easily that the two definitions of $R\left(T M^{\prime} / M, T Q\right)$ are equivalent in this case also.

Proof of C. Embed $M^{n}$ in $S^{n+k}, k$ sufficiently large so that $M^{n}$ has a normal $R^{k}$ bundle $\nu$. Now $\nu \mid D^{n} \cong D^{n} \times R^{k}$. Removing the $\operatorname{Int}\left(D^{n} \times D^{k}\right)$ form $S^{n+k}$, we get an embedding of $\left(M^{0}, \partial M^{0}\right)$ in $\left(D^{n+k}, \partial D^{n+k}\right)$ with normal bundle $\nu^{0}=\nu \mid M^{0}$, since every neighborhood of the zero section of an $R^{k}$-bundle contains an equivalent $R^{k}$-bundle (see [3]). Note that the tangent bundle of $E\left(\nu^{0}\right)$ is trivial, and $E\left(\nu^{0}\right)$ is a locally finite simplicial complex dominating $M^{0}$. Pulling $T M^{0}$ back over $E\left(\nu^{0}\right)$, we 
have that $M^{0} \times R^{n+k}$ admits a PL-manifold structure as a PL-manifold $W$ with boundary.

The Novikov-Siebenmann relative splitting theorem [1], [5], [6], provides a PL-manifold $Q^{0}$ with $\partial Q^{0}=S^{n-1}$, and a PL-homeomorphism $h: Q^{0} \times R^{n+k} \rightarrow W$. Thus $h$ defines a homotopy equivalence of pairs $\phi:\left(Q^{0}, \partial Q^{0}\right) \rightarrow\left(M^{0}, \partial M^{0}\right)$ such that $T Q^{0} \cong \phi^{*} T M^{0}$ as PL-bundles (actually stably isomorphic, but $Q^{0} \sim(n-2)$ complex, and stably isomorphic implies isomorphic). Let $\psi$ be a homotopy inverse of $\phi$; then $\psi:\left(M^{0}, \partial M^{0}\right) \rightarrow\left(Q^{0}, \partial Q^{0}\right)$ is covered by a bundle map $\psi_{*}: T M^{0}$ $\rightarrow T Q^{0}$ of topological bundles. Let $Q=Q^{0} \cup C S^{n-1}$, and $M^{\prime}=M$-point.

Then Theorem $\mathrm{A}(2)$ applies to produce an immersion in $Q$ of a neighborhood $U$ of $M^{0}$ in $M$. Thus $U$ admits a PL-manifold structure, and by Lees' lemma, $M$ admits a PL-structure. Q.E.D.

PRoof of D. Let $M$ be the underlying topological manifold of $M_{2}$, and identify it with that of $M_{1}$ via $h$. The condition on $d h$ implies by A(2), that $M \times I$ may be immersed in $M_{2} \times R$ so that the immersion is $\mathrm{PL}$ with respect to the $M_{1}$ structure near $M \times 0$ and with respect to the $M_{2}$ structure near $M \times 1$. This gives a PL-structure on $M \times I$ which is a concordance between the $M_{1}$ and $M_{2}$ structures. The result follows easily.

\section{BIBLIOGRAPHY}

1. M. A. Armstrong, Lectures on the Hauptvermutung according to Lashof and Rothenberg, Mimeographed Notes, Institute for Advanced Study, Princeton, N. J., 1968.

2. M. Brown A proof of the generalized Shoenflies theorem, Bull. Amer. Math. Soc. 65 (1960), 74-76.

3. N. Kuiper and R. Lashof, Microbundles and bundles. I, Invent. Math. 1 (1966), 1-17.

4. J. Lees, Immersions and surgeries on topological manifolds, Bull. Amer. Math. Soc. 75 (1969), 529-534.

5. S. P. Novikov, Topological invariance of rational Pontrjagin classes, Dokl. Akad. Nauk SSSR 163 (1965), 298-300=Soviet Math. Dokl. 6 (1965), 921-923.

6. L. Siebenmann, The obstruction to finding a boundary for an open manifold, Ph.D. Thesis, Princeton University, Princeton, N. J., 1965.

University of Chicago, Chicago, Illinois 60637 